\title{
Transverse oscillations of two parallel coronal loops
}

\author{
T. Van Doorsselaere ${ }^{1}$, M. S. Ruderman ${ }^{2}$, and D. Robertson ${ }^{2}$
}

\author{
${ }^{1}$ Centre for Fusion, Space and Astrophysics, Physics Department, University of Warwick, Coventry CV4 7AL, UK \\ e-mail: t.van-doorsselaere@warwick.ac.uk \\ 2 Department of Applied Mathematics, University of Sheffield, Hicks Building, Hounsfield Road, Sheffield S3 7RH, UK \\ e-mail: [m.s.ruderman; app07dr] @sheffield.ac.uk
}

Received 25 March 2008 / Accepted 28 April 2008

\begin{abstract}
Context. Collective oscillations of two or more coronal magnetic loops are observed very often.

Aims. We study the eigenmodes of oscillations of a system consisting of two parallel magnetic loops.

Methods. The linearised MHD equations for a cold plasma are solved analytically in bicylindrical coordinates using the longwavelength approximation. A dispersion equation determining the frequencies of eigenmodes is derived and solved analytically.

Results. Two solutions of the dispersion relation were found. The higher frequency corresponds to the antisymmetric mode polarised in the direction parallel to the line connecting the loop centres, and the symmetric mode polarised in the perpendicular direction. Depending on the polarisation of modes corresponding to the lower frequency, the systems of two parallel loops are classified as standard and anomalous. In standard systems the lower frequency corresponds to the symmetric mode polarised in the direction parallel to the line connecting the loop centres, and the antisymmetric mode polarised in the perpendicular direction. In anomalous systems the lower frequency corresponds to the antisymmetric mode polarised in the direction parallel to the line connecting the loop centres, and the symmetric mode polarised in the perpendicular direction. The limiting case of two identical loops is studied. The results for this case are compared with recent numerical results.
\end{abstract}

Key words. magnetohydrodynamics (MHD) - methods: analytical - Sun: corona - Sun: oscillations

\section{Introduction}

Since the first observation of transverse coronal loop oscillations by the Transition Region and Coronal Explorer (TRACE) in 1998 (Aschwanden et al. 1999; Nakariakov et al. 1999), this phenomenon is attracting ample attention from theorists. Nakariakov et al. (1999) interpreted the transverse coronal loop oscillations as fast kink oscillations of magnetic tubes. They used the simplest model of a coronal loop, which is a straight homogeneous magnetic tube. The theory of kink oscillations of such a tube was first developed by Zaitsev \& Stepanov (1975) and Ryutov \& Ryutova (1976), and then further extended by many authors (see, e.g. Edwin \& Roberts 1983). After the pioneering work by Nakariakov et al. (1999), the theorist's effort was directed into developing more realistic models of transverse coronal loop oscillations. Additional effects were incorporated in the model, e.g. the loop curvature (Van Doorsselaere et al. 2004b; Terradas et al. 2006b), the density variation along the loop (Andries et al. 2005b; Dymova \& Ruderman 2006a; Erdélyi \& Verth 2007), magnetic field twist (Ruderman 2007), and the non-circular loop cross-section (Ruderman 2003). The damping of transverse coronal loop oscillations was also extensively studied (Ruderman \& Roberts 2002; Goossens et al. 2002; Van Doorsselaere et al. 2004a; Andries et al. 2005b; Arregui et al. 2005; Dymova \& Ruderman 2006a; Terradas et al. 2006a; Terradas et al. 2006b).

The ultimate goal of building better models is to further coronal seismology and develop a tool to do remote diagnostics of physical quantities in the corona. It has been demonstrated that coronal seismology can measure the magnetic field in the corona (Nakariakov \& Ofman 2001). Recently, the presence of multiple periods in a single loop (Verwichte et al. 2004) was used to determine the density scale height (Andries et al. 2005a). This has sparked much interest, resulting in theoretical (Dymova \& Ruderman 2006a,b; McEwan et al. 2008) and observational (De Moortel \& Brady 2007; Van Doorsselaere et al. 2007) achievements.

Only last year, omnipresent wave power has been discovered in the corona (Tomczyk et al. 2007). Currently, a debate is being conducted on the interpretation of these waves (Van Doorsselaere et al. 2008; Erdélyi \& Fedun 2007) in terms of Alfvén or kink waves. When the issue will have been resolved, potentially it will be possible to use these waves for constructing magnetic field maps using coronal seismology.

Very often it is observed that not a single loop, but a whole array of loops oscillates after being perturbed by, e.g., a solar flare. Moreover, it has been suggested (see e.g. Aschwanden et al. 2000) that the loops as we see them actually consist of a multitude of individual loop strands. These considerations put on the agenda studying collective oscillations of an array of coronal loops. A natural first step in this study is the investigation of collective oscillations of a system of two coronal loops. Recently such an investigation has been carried out numerically by Luna et al. (2008). These authors considered oscillations of two identical homogeneous parallel magnetic tubes with fixed ends. They studied both the eigenmodes of oscillations of this system, and solved the initial value problem.

In this paper we aim to study the eigenmodes of a system consisting of two parallel homogeneous magnetic tubes with fixed ends. In contrast to Luna et al. (2008) we do not assume that the tubes are identical: they can have different radii and different plasma densities inside. We study the problem 


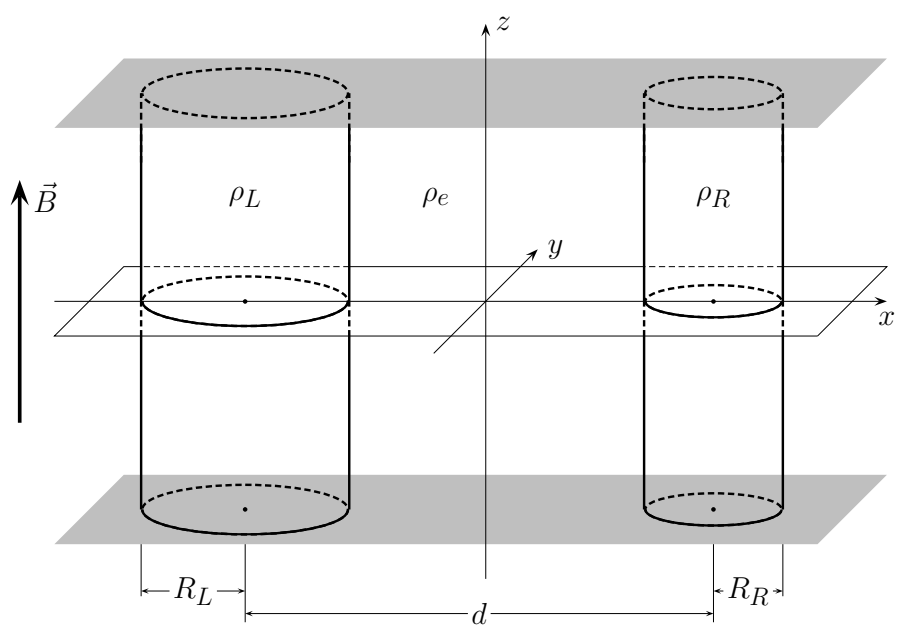

Fig. 1. Sketch of the equilibrium configuration.

analytically. To make analytical progress, we use the asymptotic theory with the ratio of the characteristic transverse scale of the system to the loop length as a small parameter. Hence, our approach is similar to the one used by Dymova \& Ruderman (2005) to derive an equation describing the transverse oscillations of a long stratified magnetic tube. The paper is organised as follows. In the next section we formulate the problem and describe bicylindrical coordinates used to study the problem. In Sect. 3 we derive the dispersion equation determining the frequencies of the eigenmodes in the long wavelength approximation and investigate the properties of the eigenmodes. In Sect. 4 we consider a particular case when the two tubes are identical. Section 5 contains the summary of the results and our conclusions.

\section{Problem formulation}

We study the eigenmodes of transverse oscillations of two parallel homogeneous magnetic loops. The loops are modelled by straight magnetic tubes of radii $R_{\mathrm{L}}$ and $R_{\mathrm{R}}$. The plasma densities inside the tubes are $\rho_{\mathrm{L}}$ and $\rho_{\mathrm{R}}$, respectively. The plasma density outside the tubes is $\rho_{\mathrm{e}}$, and it is assumed that $\rho_{\mathrm{e}}<\rho_{\mathrm{L}}, \rho_{\mathrm{R}}$. The distance between the tube axes is equal to $d>R_{\mathrm{L}}+R_{\mathrm{R}}$. The length of the tubes is $L$. It is assumed that the foot points of the magnetic loops are frozen in the dense immovable photospheric plasma, which implies that the ends of the tubes are fixed. In what follows we use Cartesian coordinates $x, y, z$ with the $z$-axis parallel to the tube axes, and the $x y$-plane cutting each of the tubes in two equal parts. The centres of the tube crosssections are on the $x$-axis. The magnetic field is assumed to be in the $z$-direction and to have constant magnitude. The equilibrium configuration is shown in Fig. 1.

We use the cold plasma approximation. This removes the slow magneto-acoustic waves from the analysis. The approximation is very good for studying fast magneto-acoustic and Alfvénic plasma oscillations in the solar corona. In this approximation the linearised MHD equations take the form

$\frac{\partial^{2} \boldsymbol{\xi}}{\partial t^{2}}=\frac{1}{\mu_{0} \rho}(\nabla \times \boldsymbol{b}) \times \boldsymbol{B}$,

$\boldsymbol{b}=\nabla \times(\boldsymbol{\xi} \times \boldsymbol{B})$,

where $\boldsymbol{\xi}$ is the plasma displacement, $\boldsymbol{b}$ is the magnetic field perturbation, $\mu_{0}$ is the magnetic permeability of free space,

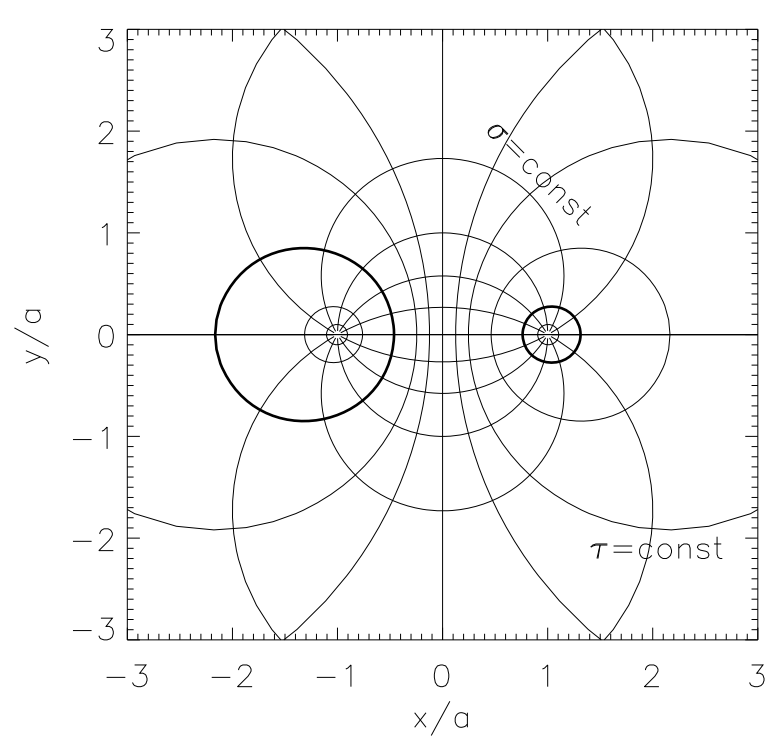

Fig. 2. Bicylindrical coordinates used in this paper. The thick circles show the tube boundaries. All coordinate lines $\sigma=$ const pass through the point $x= \pm a, y=0$. Coordinate lines $\tau=$ const are embedded circles around those points.

and $\rho$ and $\boldsymbol{B}$ are the equilibrium density and magnetic field, respectively.

To make analytical progress we use bicylindrical coordinates $\tau, \sigma, z$, where $\tau$ varies from $-\infty$ to $+\infty$, and $\sigma$ from 0 to $2 \pi$ (e.g., (Korn \& Korn 1961). The circular coordinate lines $\tau=$ const and $\sigma=$ const in the $x y$-plane are shown in Fig. 2. All coordinate lines $\sigma=$ const pass through the points $x= \pm a$ on the $x$-axis. The magnetic tube boundaries are shown by thick lines in Fig. 2. The relation between Cartesian and bicylindrical coordinates is given by

$x=\frac{a \sinh \tau}{\cosh \tau-\cos \sigma}, \quad y=\frac{a \sin \sigma}{\cosh \tau-\cos \sigma}$.

The equations of the tube boundaries are $\tau=-\tau_{\mathrm{L}}$ and $\tau=\tau_{\mathrm{R}}$. The quantities $\tau_{\mathrm{L}}$ and $\tau_{\mathrm{R}}$ are related to the tube radii by

$R_{\mathrm{L}}=\frac{a}{\sinh \tau_{\mathrm{L}}}, \quad R_{\mathrm{R}}=\frac{a}{\sinh \tau_{\mathrm{R}}}$

The inequalities $\tau<-\tau_{\mathrm{L}}$ and $\tau>\tau_{\mathrm{R}}$ determine the interiors of the left and right loop respectively, while the inequality $-\tau_{\mathrm{L}}<$ $\tau<\tau_{\mathrm{R}}$ determines the external region. The coordinates of the loop axes are $x_{\mathrm{L}}=-a \operatorname{coth} \tau_{\mathrm{L}}$ and $x_{\mathrm{R}}=a \operatorname{coth} \tau_{\mathrm{R}}$, so that

$d=a\left(\operatorname{coth} \tau_{\mathrm{L}}+\operatorname{coth} \tau_{\mathrm{R}}\right)$

The free geometrical parameters of the problem are $R_{\mathrm{L}}, R_{\mathrm{R}}$ and $d$. The quantities $\tau_{\mathrm{L}}, \tau_{\mathrm{R}}$ and $a$ are parameters of the coordinate system. It is useful to express the parameters of the coordinate system in terms of the geometrical parameters. A straightforward calculation results in

$\cosh \tau_{\mathrm{L}}=\frac{1+q^{2}\left(1-s^{2}\right)}{2 q}, \quad \cosh \tau_{\mathrm{R}}=\frac{1-q^{2}\left(1-s^{2}\right)}{2 q s}$,

where

$q=\frac{R_{\mathrm{L}}}{d}, \quad s=\frac{R_{\mathrm{R}}}{R_{\mathrm{L}}}$. 
We can always choose the coordinate system in such a way that $R_{\mathrm{R}} \leq R_{\mathrm{L}}$, so that $s \leq 1$. It follows from an obvious inequality $R_{\mathrm{L}}+R_{\mathrm{R}}<d$ that $q(1+s)<1$. It is easy to show that the righthand sides of the two equations in (6) are larger than unity, so that we can always find $\tau_{\mathrm{L}}$ and $\tau_{\mathrm{R}}$ satisfying these equations.

The parameter $a$ can be determined by using these expressions for $\tau_{\mathrm{R}, \mathrm{L}}$ and Eq. (4).

In what follows we also use the expressions for the gradient and the Laplace operator,

$$
\begin{aligned}
& \nabla f=\frac{\cosh \tau-\cos \sigma}{a}\left(\boldsymbol{e}_{\tau} \frac{\partial f}{\partial \tau}+\boldsymbol{e}_{\sigma} \frac{\partial f}{\partial \sigma}\right)+\boldsymbol{e}_{z} \frac{\partial f}{\partial z}, \\
& \nabla^{2} f=\frac{(\cosh \tau-\cos \sigma)^{2}}{a^{2}}\left(\frac{\partial^{2} f}{\partial \tau^{2}}+\frac{\partial^{2} f}{\partial \sigma^{2}}\right)+\frac{\partial^{2} f}{\partial z^{2}},
\end{aligned}
$$

where $f$ is an arbitrary function, and $\boldsymbol{e}_{\tau}, \boldsymbol{e}_{\sigma}$ and $\boldsymbol{e}_{z}$ are the unit vectors in the $\tau, \sigma$ and $z$-direction respectively.

It follows from Eq. (3) that $x \rightarrow-a$ and $y \rightarrow 0$ as $\tau \rightarrow-\infty$, and $x \rightarrow a$ and $y \rightarrow 0$ as $\tau \rightarrow \infty$. These relations imply that the solution to the system of Eqs. (1) and (2) has to be bounded as $\tau \rightarrow \pm \infty$.

Let us introduce $r=\sqrt{x^{2}+y^{2}}$. It is straightforward to show that $\tau \rightarrow 0$ and $\sigma \rightarrow 0$ as $r \rightarrow \infty$.

The condition that the magnetic field lines are frozen in the dense plasma at the ends of the magnetic tubes reads

$\xi=0, \quad$ at $z= \pm L / 2$.

The magnetic pressure, $P$, and the normal component of the displacement, $\xi_{\tau}$, has to be continuous at the tube boundaries. Let us introduce the jump of function $f(\tau)$ across the tube boundary,

$[f]=\lim _{\varepsilon \rightarrow+0}\left\{f\left(\tau_{0}+\varepsilon\right)-f\left(\tau_{0}-\varepsilon\right)\right\}$,

where either $\tau_{0}=-\tau_{\mathrm{L}}$, or $\tau_{0}=\tau_{\mathrm{R}}$. Then the boundary conditions at the tube boundaries are written as

$[P]=0, \quad\left[\xi_{\tau}\right]=0, \quad$ at $\tau=-\tau_{\mathrm{L}}, \tau_{\mathrm{R}}$.

In addition, the solution has to vanish at infinity, i.e. as $r \rightarrow \infty$.

The system of Eqs. (1) and (2) together with the boundary conditions (11), conditions of regularity as $\tau \rightarrow \pm \infty$, and the vanishing conditions at infinity will be used in the next section to study the eigenmodes of the two-tube system.

\section{Eigenmodes}

In this section we are looking for the eigenmodes of the twotube system. For this we first derive from Eqs. (1) and (2) the system of equations for $P$ and $\boldsymbol{\xi}$. It follows from (1) that $\xi_{z}=0$. In the linear approximation the magnetic pressure is given by $P=B b_{z} / \mu_{0}$. Equation (2) can be rewritten as

$\boldsymbol{b}=B \frac{\partial \boldsymbol{\xi}}{\partial z}-\boldsymbol{e}_{z} B \nabla \cdot \boldsymbol{\xi}$

It follows from this equation that

$P=-\rho V_{\mathrm{A}}^{2} \nabla \cdot \boldsymbol{\xi}$,

where the square of the Alfvén speed is given by

$V_{\mathrm{A}}^{2}=\frac{B^{2}}{\mu_{0} \rho}$.
Now we substitute (12) in (1) and use (13) to obtain

$\frac{\partial^{2} \boldsymbol{\xi}}{\partial t^{2}}=V_{\mathrm{A}}^{2} \frac{\partial^{2} \boldsymbol{\xi}}{\partial z^{2}}-\frac{1}{\rho} \nabla_{\perp} P$,

where $\nabla_{\perp} f$ is given by

$\nabla_{\perp} f=\nabla f-\boldsymbol{e}_{z} \frac{\partial f}{\partial z}$

Eliminating $\boldsymbol{\xi}$ from (13) and (15) we obtain the wave equation for $P$,

$\frac{\partial^{2} P}{\partial t^{2}}-V_{\mathrm{A}}^{2} \nabla^{2} P=0$

Writing Eq. (15) in components yields

$\frac{\partial^{2} \xi_{\tau}}{\partial t^{2}}-V_{\mathrm{A}}^{2} \frac{\partial^{2} \xi_{\tau}}{\partial z^{2}}=-\frac{\cosh \tau-\cos \sigma}{a \rho} \frac{\partial P}{\partial \tau}$

$\frac{\partial^{2} \xi_{\sigma}}{\partial t^{2}}-V_{\mathrm{A}}^{2} \frac{\partial^{2} \xi_{\sigma}}{\partial z^{2}}=-\frac{\cosh \tau-\cos \sigma}{a \rho} \frac{\partial P}{\partial \sigma}$

Now we look for the solution to the system of Eqs. (17)-(19) and the boundary conditions (11) in the form of eigenmodes, so that we take all the variables proportional to $\exp (-i \omega t)$. To satisfy the boundary conditions (10) we take $\boldsymbol{\xi}$ proportional to $\cos (\pi z / L)$. Then it follows from (13) that $P$ is also proportional to $\cos (\pi z / L)$. After that Eqs. (17)-(19) reduce to

$\left(\omega^{2}-V_{\mathrm{A}}^{2} k^{2}\right) P+V_{\mathrm{A}}^{2} \nabla_{\perp}^{2} P=0$,

$\left(\omega^{2}-V_{\mathrm{A}}^{2} k^{2}\right) \xi_{\tau}=\frac{\cosh \tau-\cos \sigma}{a \rho} \frac{\partial P}{\partial \tau}$

$\left(\omega^{2}-V_{\mathrm{A}}^{2} k^{2}\right) \xi_{\sigma}=\frac{\cosh \tau-\cos \sigma}{a \rho} \frac{\partial P}{\partial \sigma}$,

where

$k=\frac{\pi}{L}, \quad \nabla_{\perp}^{2}=\frac{(\cosh \tau-\cos \sigma)^{2}}{a^{2}}\left(\frac{\partial^{2}}{\partial \tau^{2}}+\frac{\partial^{2}}{\partial \sigma^{2}}\right)$.

Now we assume that the loop length is much larger than the size of the system in the transverse direction. This condition can be written as $\epsilon=d / L \ll 1$. It follows from Eq. (5) that $a<d$. However, we do not assume that $a$ and $d$ are of the same order. Our method works both for $a \sim d$ and for $a \ll d$. We can thus see that $k a \lesssim \epsilon$. In what follows we are looking for eigenmodes with the frequencies of the order of $k V_{\mathrm{A}}$. This implies that $\omega a / V_{\mathrm{A}} \lesssim \epsilon$, so that the ratio of the first term in Eq. (20) to the second one is of the order of $\epsilon^{2}$. In what follows we are looking for the solution to the problem in the lowest order approximation with respect to $\epsilon$. Then we can neglect the first term in Eq. (20) and rewrite it in a simplified form as

$\frac{\partial^{2} P}{\partial \tau^{2}}+\frac{\partial^{2} P}{\partial \sigma^{2}}=0$

By separation of variables, we find a solution of the form

$P=\Phi(\tau) \cos \left[m\left(\sigma-\sigma_{0}\right)\right]-\Phi(0) \cos \left(m \sigma_{0}\right)$,

where the separation constant $m$ is a positive integer and $\sigma_{0}$ is an integration constant. The second term in this expression is added to ensure that $P \rightarrow 0$ as $r \rightarrow \infty$, which is equivalent to $\tau \rightarrow 0$ and $\sigma \rightarrow 0$. Substituting Eq. (25) in Eq. (24) we obtain

$\Phi=C_{1} \mathrm{e}^{m \tau}+C_{2} \mathrm{e}^{-m \tau}$, 
where $C_{1}$ and $C_{2}$ are arbitrary integration constants. Taking into account the regularity conditions at $\tau \rightarrow \pm \infty$ yields

$\Phi= \begin{cases}C_{\mathrm{L}} \mathrm{e}^{m \tau}, & \tau<-\tau_{\mathrm{L}}, \\ C_{1} \mathrm{e}^{m \tau}+C_{2} \mathrm{e}^{-m \tau}, & -\tau_{\mathrm{L}}<\tau<\tau_{\mathrm{R}}, \\ C_{\mathrm{R}} \mathrm{e}^{-m \tau}, & \tau>\tau_{\mathrm{R}},\end{cases}$

where once again $C_{\mathrm{L}}$ and $C_{\mathrm{R}}$ are arbitrary constants. Using Eq. (21) we obtain $\xi_{\tau}=\hat{\xi}_{\tau} \cos \left[m\left(\sigma-\sigma_{0}\right)\right]$, where

$\hat{\xi}_{\tau}=\frac{m(\cosh \tau-\cos \sigma)}{a}\left\{\begin{array}{l}\frac{C_{\mathrm{L}} \mathrm{e}^{m \tau}}{\rho_{\mathrm{L}}\left(\omega^{2}-V_{\mathrm{AL}}^{2} k^{2}\right)}, \tau<-\tau_{\mathrm{L}}, \\ \frac{C_{1} \mathrm{e}^{m \tau}-C_{2} \mathrm{e}^{-m \tau}}{\rho_{\mathrm{e}}\left(\omega^{2}-V_{\mathrm{Ae}}^{2} k^{2}\right)},-\tau_{\mathrm{L}}<\tau<\tau_{\mathrm{R}}, \\ \frac{-C_{\mathrm{R}} \mathrm{e}^{-m \tau}}{\rho_{\mathrm{R}}\left(\omega^{2}-V_{\mathrm{AR}}^{2} k^{2}\right)}, \tau>\tau_{\mathrm{R}} .\end{array}\right.$

Substituting Eqs. (27)-(28) in Eq. (11) gives the system of linear homogeneous algebraic equations for $C_{1}, C_{2}, C_{\mathrm{L}}$ and $C_{\mathrm{R}}$,

$$
\begin{aligned}
& C_{1} \mathrm{e}^{-m \tau_{\mathrm{L}}}+C_{2} \mathrm{e}^{m \tau_{\mathrm{L}}}=C_{\mathrm{L}} \mathrm{e}^{-m \tau_{\mathrm{L}}}, \\
& C_{1} \mathrm{e}^{m \tau_{\mathrm{R}}}+C_{2} \mathrm{e}^{-m \tau_{\mathrm{R}}}=C_{\mathrm{R}} \mathrm{e}^{-m \tau_{\mathrm{R}}}, \\
& \frac{C_{1} \mathrm{e}^{-m \tau_{\mathrm{L}}}-C_{2} \mathrm{e}^{m \tau_{\mathrm{L}}}}{\rho_{\mathrm{e}}\left(\omega^{2}-V_{\mathrm{Ae}}^{2} k^{2}\right)}=\frac{C_{\mathrm{L}} \mathrm{e}^{-m \tau_{\mathrm{L}}}}{\rho_{\mathrm{L}}\left(\omega^{2}-V_{\mathrm{AL}}^{2} k^{2}\right)}, \\
& \frac{C_{1} \mathrm{e}^{m \tau_{\mathrm{R}}}-C_{2} \mathrm{e}^{-m \tau_{\mathrm{R}}}}{\rho_{\mathrm{e}}\left(\omega^{2}-V_{\mathrm{Ae}}^{2} k^{2}\right)}=\frac{-C_{\mathrm{R}} \mathrm{e}^{-m \tau_{\mathrm{R}}}}{\rho_{\mathrm{R}}\left(\omega^{2}-V_{\mathrm{AR}}^{2} k^{2}\right)} .
\end{aligned}
$$

This system of equations has a non-trivial solution only when its determinant is zero. This condition results in the dispersion equation,

$$
\begin{gathered}
{\left[\left(\rho_{\mathrm{L}}+\rho_{\mathrm{e}}\right) \omega^{2}-2 \rho V_{\mathrm{A}}^{2} k^{2}\right]\left[\left(\rho_{\mathrm{R}}+\rho_{\mathrm{e}}\right) \omega^{2}-2 \rho V_{\mathrm{A}}^{2} k^{2}\right]} \\
-\omega^{4}\left(\rho_{\mathrm{L}}-\rho_{\mathrm{e}}\right)\left(\rho_{\mathrm{R}}-\rho_{\mathrm{e}}\right) E^{2}=0,
\end{gathered}
$$

where $\rho V_{\mathrm{A}}^{2}=\rho_{\mathrm{L}} V_{\mathrm{AL}}^{2}=\rho_{\mathrm{R}} V_{\mathrm{AR}}^{2}=\rho_{\mathrm{e}} V_{\mathrm{Ae}}^{2}=B^{2} / \mu_{0}$, and $E=$ $\exp \left[-m\left(\tau_{\mathrm{L}}+\tau_{\mathrm{R}}\right)\right]$. The solutions to this dispersion equation are

$$
\begin{aligned}
\omega_{ \pm}^{2} & =\frac{V_{\mathrm{Ae}}^{2} k^{2}}{\left(\zeta_{\mathrm{L}}+1\right)\left(\zeta_{\mathrm{R}}+1\right)-\left(\zeta_{\mathrm{L}}-1\right)\left(\zeta_{\mathrm{R}}-1\right) E^{2}} \\
& \times\left\{\zeta_{\mathrm{L}}+\zeta_{\mathrm{R}}+2 \pm \sqrt{\left(\zeta_{\mathrm{L}}-\zeta_{\mathrm{R}}\right)^{2}+4\left(\zeta_{\mathrm{L}}-1\right)\left(\zeta_{\mathrm{R}}-1\right) E^{2}}\right\},
\end{aligned}
$$

where

$\zeta_{\mathrm{L}}=\frac{\rho_{\mathrm{L}}}{\rho_{\mathrm{e}}}, \quad \zeta_{\mathrm{R}}=\frac{\rho_{\mathrm{R}}}{\rho_{\mathrm{e}}}$.

In the case of one vanishing loop, we take $\rho_{\mathrm{L}}=\rho_{\mathrm{e}}$. In that case, Eq. (31) reduces to

$\omega^{2}=\frac{2 \rho V_{\mathrm{A}}^{2} k^{2}}{\rho_{\mathrm{e}}+\rho_{\mathrm{R}}} \quad$ and $\omega^{2}=V_{\mathrm{Ae}}^{2} k^{2}$,

correctly recovering the kink frequency for the right loop.

We can immediately notice one difference between the double-tube system and a single tube. The eigenfrequencies $\omega_{ \pm}$ depend on $m$, while in the case of a single tube the eigenfrequencies are equal to $\omega_{k}=c_{k} k$ for all $m>0$ in the long-wavelength limit, where $c_{k}$ is the kink speed.

Let us now study the eigenmode polarisation. For this we calculate the $x$ and $y$-component of the plasma displacement, $\xi_{x}$ and $\xi_{y}$. We start our analysis from expressing the unit vectors of bicylindrical coordinates, $\boldsymbol{e}_{\tau}$ and $\boldsymbol{e}_{\sigma}$, in terms of the unit vectors of Cartesian coordinates, $\boldsymbol{e}_{x}$ and $\boldsymbol{e}_{y}$. Let us introduce the position vector $\boldsymbol{r}=x \boldsymbol{e}_{x}+y \boldsymbol{e}_{y}$. We know that $\boldsymbol{e}_{\tau}$ is parallel to $\partial \boldsymbol{r} / \partial \tau$, and $\boldsymbol{e}_{\sigma}$ is parallel to $\partial \boldsymbol{r} / \partial \sigma$. Using this fact we easily obtain

$$
\begin{aligned}
\boldsymbol{e}_{\tau} & =\frac{\boldsymbol{e}_{x}(1-\cosh \tau \cos \sigma)-\boldsymbol{e}_{y} \sinh \tau \sin \sigma}{\cosh \tau-\cos \sigma} \\
\boldsymbol{e}_{\sigma} & =-\frac{\boldsymbol{e}_{x} \sinh \tau \sin \sigma+\boldsymbol{e}_{y}(1-\cosh \tau \cos \sigma)}{\cosh \tau-\cos \sigma} .
\end{aligned}
$$

The next step is to find the expressions for $\xi_{\tau}$ and $\xi_{\sigma}$. Using Eqs. (21), (22) and (25) yields

$$
\begin{aligned}
& \xi_{\tau}=\frac{(\cosh \tau-\cos \sigma) \cos \left[m\left(\sigma-\sigma_{0}\right)\right]}{a \rho\left(\omega^{2}-V_{\mathrm{A}}^{2} k^{2}\right)} \Phi^{\prime}(\tau), \\
& \xi_{\sigma}=-\frac{m(\cosh \tau-\cos \sigma) \sin \left[m\left(\sigma-\sigma_{0}\right)\right]}{a \rho\left(\omega^{2}-V_{\mathrm{A}}^{2} k^{2}\right)} \Phi(\tau),
\end{aligned}
$$

where the prime indicates the derivative. It follows from Eq. (33) that

$$
\begin{aligned}
& \xi_{x}=\frac{\xi_{\tau}(1-\cosh \tau \cos \sigma)-\xi_{\sigma} \sinh \tau \sin \sigma}{\cosh \tau-\cos \sigma}, \\
& \xi_{y}=-\frac{\xi_{\tau} \sinh \tau \sin \sigma+\xi_{\sigma}(1-\cosh \tau \cos \sigma)}{\cosh \tau-\cos \sigma} .
\end{aligned}
$$

Substituting Eq. (34) in Eq. (35) we arrive at

$$
\begin{aligned}
\xi_{x}= & \frac{1}{a \rho\left(\omega^{2}-V_{\mathrm{A}}^{2} k^{2}\right)}\left\{\Phi^{\prime}(1-\cosh \tau \cos \sigma) \cos \left[m\left(\sigma-\sigma_{0}\right)\right]\right. \\
& \left.+m \Phi \sinh \tau \sin \sigma \sin \left[m\left(\sigma-\sigma_{0}\right)\right]\right\}
\end{aligned}
$$

$$
\begin{aligned}
\xi_{y}= & -\frac{1}{a \rho\left(\omega^{2}-V_{\mathrm{A}}^{2} k^{2}\right)}\left\{\Phi^{\prime} \sinh \tau \sin \sigma \cos \left[m\left(\sigma-\sigma_{0}\right)\right]\right. \\
& \left.-m \Phi(1-\cosh \tau \cos \sigma) \sin \left[m\left(\sigma-\sigma_{0}\right)\right]\right\} .
\end{aligned}
$$

In what follows, we concentrate on the cases $\sigma_{0}=0$ and $\sigma_{0}=\pi / 2$. As will be shown, the value of $\sigma_{0}$ determines the polarisation of oscillation. The two cases $\sigma_{0}=0$ and $\sigma_{0}=\pi / 2$ are the two extremes, and have a polarisation perpendicular to each other. Any other value of $\sigma_{0}$ is intermediate between these two extremes.

It follows from Eqs. (36) and (37) that $\xi_{x}$ is an even and $\xi_{y}$ an odd function of $\sigma$ when $\sigma_{0}=0$, which implies that the displacement is symmetric with respect to the $x$-axis. In particular, the displacement of points at the $x$-axis, corresponding to $\sigma=0$, is in the $x$-direction. When $m=1$, these wave modes correspond to the $S_{x}$ and $A_{x}$ modes found numerically by Luna et al. (2008), where $S$ and $A$ stand for symmetric and antisymmetric, and the subscript indicates the direction of polarisation.

When $\sigma_{0}=\pi / 2$ and $m$ is odd, $\xi_{x}$ is an odd and $\xi_{y}$ an even function of $\sigma$. For the system of two identical loops $\left(R_{\mathrm{L}}=R_{\mathrm{R}}\right)$ this implies that the displacement is symmetric with respect to the $y$-axis. When $m=1$, these wave modes correspond to the $S_{y}$ and $A_{y}$ modes found numerically by Luna et al. (2008).

The expressions for the displacement components become especially simple when $\tau \rightarrow-\infty$ or $\tau \rightarrow \infty$, which correspond to $y=0$ and $x= \pm a$. Using Eqs. (27) and (36) it is straightforward to obtain

$$
\begin{aligned}
& \xi_{x L}=\frac{-C_{\mathrm{L}} \cos \sigma_{0}}{2 a \rho_{\mathrm{L}}\left(\omega^{2}-V_{\mathrm{AL}}^{2} k^{2}\right)}, \quad \xi_{y L}=\frac{C_{\mathrm{L}} \sin \sigma_{0}}{2 a \rho_{\mathrm{L}}\left(\omega^{2}-V_{\mathrm{AL}}^{2} k^{2}\right)} \\
& \xi_{x R}=\frac{C_{\mathrm{R}} \cos \sigma_{0}}{2 a \rho_{\mathrm{R}}\left(\omega^{2}-V_{\mathrm{AR}}^{2} k^{2}\right)}, \quad \xi_{y R}=\frac{C_{\mathrm{R}} \sin \sigma_{0}}{2 a \rho_{\mathrm{R}}\left(\omega^{2}-V_{\mathrm{AR}}^{2} k^{2}\right)}
\end{aligned}
$$


when $m=1$, while

$\xi_{x \mathrm{~L}}=\xi_{y \mathrm{~L}}=\xi_{x \mathrm{R}}=\xi_{y \mathrm{R}}=0$

when $m>1$, where $\xi_{x, y \mathrm{~L}}=\lim _{\tau \rightarrow-\infty} \xi_{x, y}$ and $\xi_{x, y \mathrm{R}}=\lim _{\tau \rightarrow \infty} \xi_{x, y}$. Eq. (40) implies that the modes with $m>1$ do not displace the points $x= \pm a$. This property is similar to the property of fluting modes in a single magnetic tube which do not displace the tube axes, although we have to note that the points $x= \pm a$ are close to the tube axes only when the tubes are sufficiently far from each other, i.e. when $d \gg R_{\mathrm{L}}+R_{\mathrm{R}}$.

Using the first two equations in Eq. (29) to express $C_{1}$ and $C_{2}$ in terms of $C_{\mathrm{L}}$ and $C_{\mathrm{R}}$, substituting the results in the third equation in Eq. (29), and using the dispersion equation, Eq. (30), we obtain that the ratio of $C_{\mathrm{L}}$ and $C_{\mathrm{R}}$ is given by

$$
\frac{C_{\mathrm{R}}}{C_{\mathrm{L}}}=\mathrm{e}^{2 m \tau_{\mathrm{R}}} \frac{\left(\zeta_{\mathrm{L}}+1\right) \omega^{2}-2 V_{\mathrm{Ae}}^{2} k^{2}}{\left(\zeta_{\mathrm{R}}-1\right) \omega^{2}} \frac{\zeta_{\mathrm{R}} \omega^{2}-V_{\mathrm{Ae}}^{2} k^{2}}{\zeta_{\mathrm{L}} \omega^{2}-V_{\mathrm{Ae}}^{2} k^{2}} .
$$

In what follows we concentrate on the case of $m=1$. This is the only case for which the axes of the tubes are displaced (see Eq. (39), (40)), and in that respect they resemble the kink modes of the classical cylindrical model.

Let us introduce the function

$$
f(X)=\left[\left(\zeta_{\mathrm{L}}+1\right) X-2\right]\left[\left(\zeta_{\mathrm{R}}+1\right) X-2\right]-\left(\zeta_{\mathrm{L}}-1\right)\left(\zeta_{\mathrm{R}}-1\right) X^{2} E^{2} .
$$

This function is equal to the left-hand side of Eq. (30) divided by $\left(\rho V_{\mathrm{A}}^{2} k^{2}\right)^{2}$, and with $X$ substituted for $\omega^{2} / V_{\mathrm{Ae}}^{2} k^{2}$. Function $f(X)$ is a quadratic polynomial with the first coefficient positive. Its zeros are $X_{ \pm}=\omega_{ \pm}^{2} / V_{\mathrm{Ae}}^{2} k^{2}$. Since $f\left(2\left(\zeta_{\mathrm{L}, \mathrm{R}}+1\right)^{-1}\right)<0$, it follows that $X_{-}<2\left(\zeta_{\mathrm{L}, \mathrm{R}}+1\right)^{-1}<X_{+}$. This implies that the following two inequalities are satisfied,

$\left(\zeta_{\mathrm{L}, \mathrm{R}}+1\right) \omega_{+}^{2}-2 V_{\mathrm{Ae}}^{2} k^{2}>0$,

$\left(\zeta_{\mathrm{L}, \mathrm{R}}+1\right) \omega_{-}^{2}-2 V_{\mathrm{Ae}}^{2} k^{2}<0$.

Since $2 \zeta_{\mathrm{L}, \mathrm{R}} \omega_{+}^{2}>\left(\zeta_{\mathrm{L}, \mathrm{R}}+1\right) \omega_{+}^{2}$, it follows from Eq. (43) that

$\zeta_{\mathrm{L}} \omega_{+}^{2}-V_{\mathrm{Ae}}^{2} k^{2}>0, \quad \zeta_{\mathrm{R}} \omega_{+}^{2}-V_{\mathrm{Ae}}^{2} k^{2}>0$.

Equations (43) and (45) together with Eq. (41) imply that $C_{\mathrm{R}}$ and $C_{\mathrm{L}}$ have the same sign when $\omega=\omega_{+}$. Then it follows from Eqs. (38) and (39) that, when $\omega=\omega_{+}$, the two tubes are displaced in opposite directions parallel to the $x$-axis when $\sigma_{0}=0$, while they are displaced in the same directions parallel to the $y$-axis when $\sigma_{0}=\pi / 2$. This means that $\omega_{+}$is the frequency of the $A_{x}$ and $S_{y}$ modes. This result is in qualitative agreement with the numerical results by Luna et al. (2008) who found that the frequencies of the $A_{x}$ and $S_{y}$ modes are larger than the frequencies of the $S_{x}$ and $A_{y}$ modes.

The situation with the low-frequency modes is more complicated. To study the properties of these modes we first note that, in accordance with Eq. (45), $\zeta_{\mathrm{L}, \mathrm{R}}^{-1}<X_{+}$. Then it follows that $\zeta_{\mathrm{L}}^{-1}>X_{-}$or, what is the same, $\zeta_{\mathrm{L}} \omega_{-}^{2}-V_{\mathrm{Ae}}^{2} k^{2}>0$, if $f\left(\zeta_{\mathrm{L}}^{-1}\right)<0$. This condition can be written as

$2 \zeta_{\mathrm{L}}<\zeta_{\mathrm{R}}+1+\left(\zeta_{\mathrm{R}}-1\right) E^{2}$.

Similarly we obtain that $\zeta_{\mathrm{R}} \omega_{-}^{2}-V_{\mathrm{Ae}}^{2} k^{2}>0$ if

$2 \zeta_{\mathrm{R}}<\zeta_{\mathrm{L}}+1+\left(\zeta_{\mathrm{L}}-1\right) E^{2}$

Hence, if both inequalities, Eqs. (46) and (47), are not satisfied, then $\zeta_{\mathrm{L}, \mathrm{R}} \omega_{-}^{2}-V_{\mathrm{Ae}}^{2} k^{2}<0$. This result together with Eqs. (41)

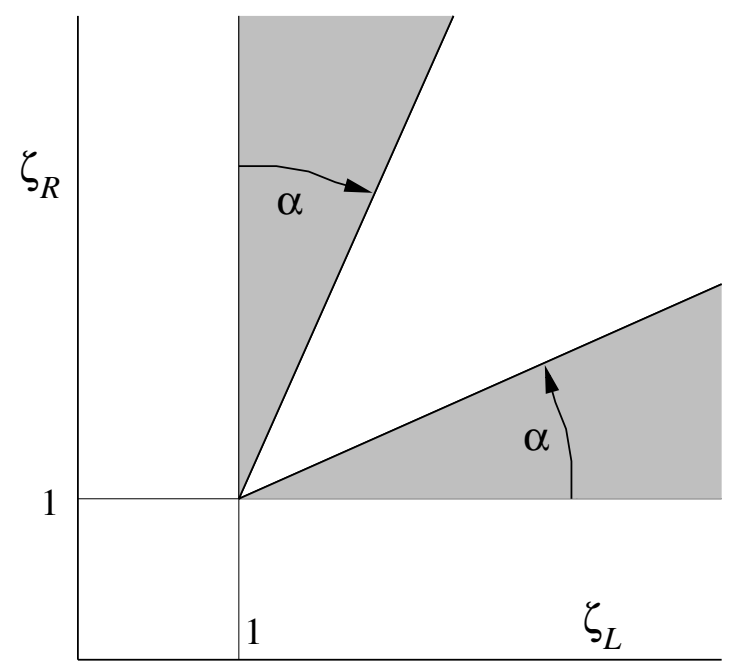

Fig. 3. The regions corresponding to standard and anomalous systems in the $\zeta_{L} \zeta_{R}$-plane for a fixed value of $E$. The two shaded sectors correspond to systems with the anomalous behaviour, while the non-shaded sector to systems with the standard behaviour (recall that the admissible region of parameters is $\zeta_{L}>1, \zeta_{R}>1$ ). The inequality (46) is satisfied in the upper shaded region, and the inequality (47) is satisfied in the lower shaded region.

and (44) implies that $C_{\mathrm{R}}$ and $C_{\mathrm{L}}$ have opposite signs when $\omega=\omega_{-}$. This means that $\omega_{-}$is the frequency of the $S_{x}$ and $A_{y}$ modes. Once again this result is in qualitative agreement with the numerical results by Luna et al. (2008) who found that the frequencies of the $S_{x}$ and $A_{y}$ modes are lower than the frequencies of the $A_{x}$ and $S_{y}$ modes. In what follows we will call systems with such properties of the low-frequency kink modes standard, or systems with standard behaviour.

Let us now assume that one of the two inequalities, Eqs. (46) and (47), is satisfied (it is easy to see that they cannot be satisfied simultaneously). In this case $\zeta_{\mathrm{L}} \omega_{-}^{2}-V_{\mathrm{Ae}}^{2} k^{2}$ and $\zeta_{\mathrm{R}} \omega_{-}^{2}-V_{\mathrm{Ae}}^{2} k^{2}$ have opposite signs. Together with Eqs. (41) and (44) this result implies that $C_{\mathrm{R}}$ and $C_{\mathrm{L}}$ have the same sign when $\omega=\omega_{-}$. This means that $\omega_{-}$is the frequency of the $A_{x}$ and $S_{y}$ modes. We see that now there are two $A_{x}$ modes, high- and low-frequency, and also two $S_{y}$ modes, high- and low-frequency, while there are no $S_{x}$ and $A_{y}$ modes. In what follows we will call systems with such properties of the kink modes anomalous, or systems with anomalous behaviour. In Fig. 3 the regions in the $\zeta_{\mathrm{L}} \zeta_{\mathrm{R}^{-}}$ plane corresponding to systems with standard and anomalous behaviour are shown for a fixed value of $E$ (or, what is the same, for a fixed value of $\tau_{\mathrm{L}}+\tau_{\mathrm{R}}$ ). The angle $\alpha$ in Fig. 3 is given by

$\alpha=\arctan \frac{1+E^{2}}{2}<\frac{\pi}{4}$.

We see that the line $\zeta_{\mathrm{L}}=\zeta_{\mathrm{R}}$ is always inside the region corresponding to the standard behaviour. Since Luna et al. (2008) studied oscillations of a system of two identical loops, it is not surprising that they did not find the anomalous behaviour.

We see that the properties of standard systems are qualitatively in agreement with the numerical results by Luna et al. (2008). Quantitatively, however, we found that the frequencies of the $A_{x}$ and $S_{y}$ modes are equal, and the same is true for $S_{x}$ and $A_{y}$ modes. Luna et al. (2008) found that the frequency of the $A_{x}$ mode is larger than the frequency of the $S_{y}$ mode, while the frequency of the $A_{y}$ mode is larger than the frequency of the $S_{x}$ mode, although the difference between the two latter frequencies is very small. We discuss this difference between the analytical 


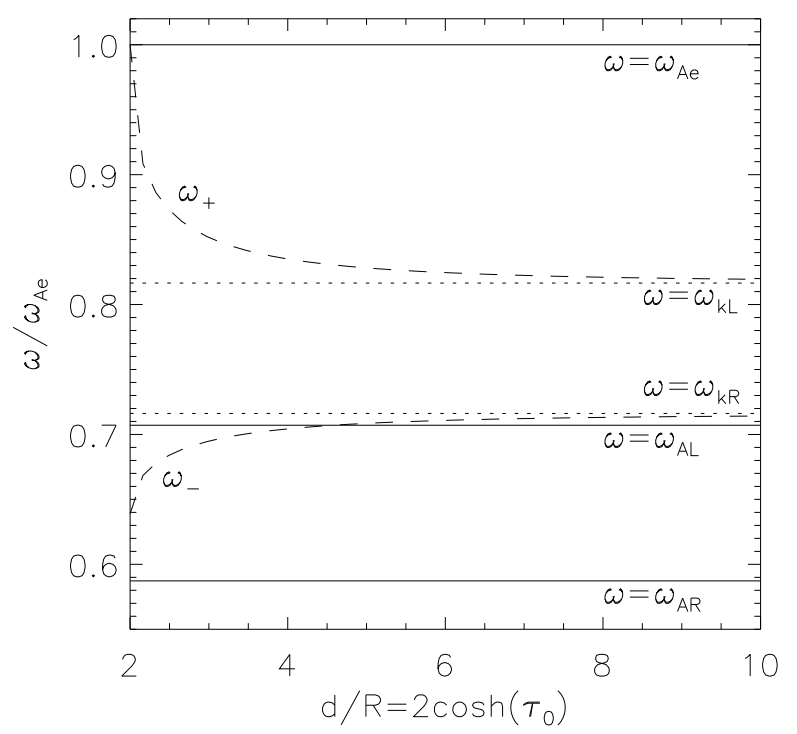

Fig. 4. The eigenfrequencies for $\zeta_{\mathrm{L}}=2$ and $\zeta_{\mathrm{R}}=2.9$ versus the separation between loops (dashed lines). The internal $\left(\omega_{\mathrm{AL}, \mathrm{R}}\right)$ and external $\left(\omega_{\mathrm{Ae}}\right)$ cut-off frequencies are plotted with full lines. The kink frequencies of the separate tubes $\left(\omega_{\mathrm{kL}, \mathrm{R}}\right)$ are plotted with dotted lines.

and numerical results in more detail in Sect. 4 where we consider a particular case of two identical tubes.

Let us now calculate the limiting values of $\omega_{+}$and $\omega_{-}$when the separation between the loops is large. To do this we formally consider the limits as $d \rightarrow \infty$, or, which is same $a \rightarrow \infty$. Then it follows from equation (4) that $\tau_{\mathrm{L}}, \tau_{\mathrm{R}} \rightarrow \infty$, so that $E \rightarrow 0$. After that we obtain from Eq. (31) that

$$
\begin{aligned}
& \omega_{+} \rightarrow \omega_{0+}=\max \left(c_{k \mathrm{~L}} k, c_{k \mathrm{R}} k\right), \\
& \omega_{-} \rightarrow \omega_{0-}=\min \left(c_{k \mathrm{~L}} k, c_{k \mathrm{R}} k\right),
\end{aligned}
$$

where $c_{k \mathrm{~L}}$ and $c_{k \mathrm{R}}$ are the kink speeds of the left and right tubes determined by

$c_{k \mathrm{~L}}^{2}=\frac{2 \rho V_{\mathrm{A}}^{2}}{\rho_{\mathrm{e}}+\rho_{\mathrm{L}}}, \quad c_{k \mathrm{R}}^{2}=\frac{2 \rho V_{\mathrm{A}}^{2}}{\rho_{\mathrm{e}}+\rho_{\mathrm{R}}}$.

In particular, when the two tubes are identical, both $\omega_{+}$and $\omega_{-}$ tend to the common kink frequency of the two tubes, $\omega_{k}=c_{k} k=$ $c_{k \mathrm{~L}} k=c_{k \mathrm{R}} k$. This result is in an excellent agreement with Luna et al. (2008) who found that the frequencies of the four modes, $S_{x}, A_{x}, S_{y}$ and $A_{y}$, tend to $\omega_{k}$ as $d \rightarrow \infty$.

It is now possible to gain a physical understanding of anomalous systems. To do that we look at the internal cut-off frequencies for the left $\left(\omega_{\mathrm{AL}}\right)$ and right tube $\left(\omega_{\mathrm{AR}}\right)$ separately, the external cut-off frequency $\left(\omega_{\mathrm{Ae}}\right)$, and the kink frequencies of each tube serarately $\left(\omega_{k \mathrm{~L}, \mathrm{R}}\right)$. For simplicity, we take $\tau_{\mathrm{L}}=\tau_{\mathrm{R}}=\tau_{0}$. A plot of the eigenfrequencies of the system with $\zeta_{\mathrm{L}}=2$ and $\zeta_{\mathrm{R}}=2.9$ is presented in Fig. 4. We see that, as $d / R \rightarrow \infty$, the eigenfrequencies tend to the separate kink frequencies. We also see that, when $d / R$ decreases, $\omega_{-}$drops below the internal cut-off frequency. That is the point when the systems becomes anomalous. The left tube cannot support the global, fast magnetosoniclike oscillation, because the frequency of the collective oscillation is below its internal Alfvén frequency, which is a cut-off for fast magnetosonic oscillations.

This can also be observed when looking at the eigenfunctions (see Fig. 5). For this figure, we have taken the same
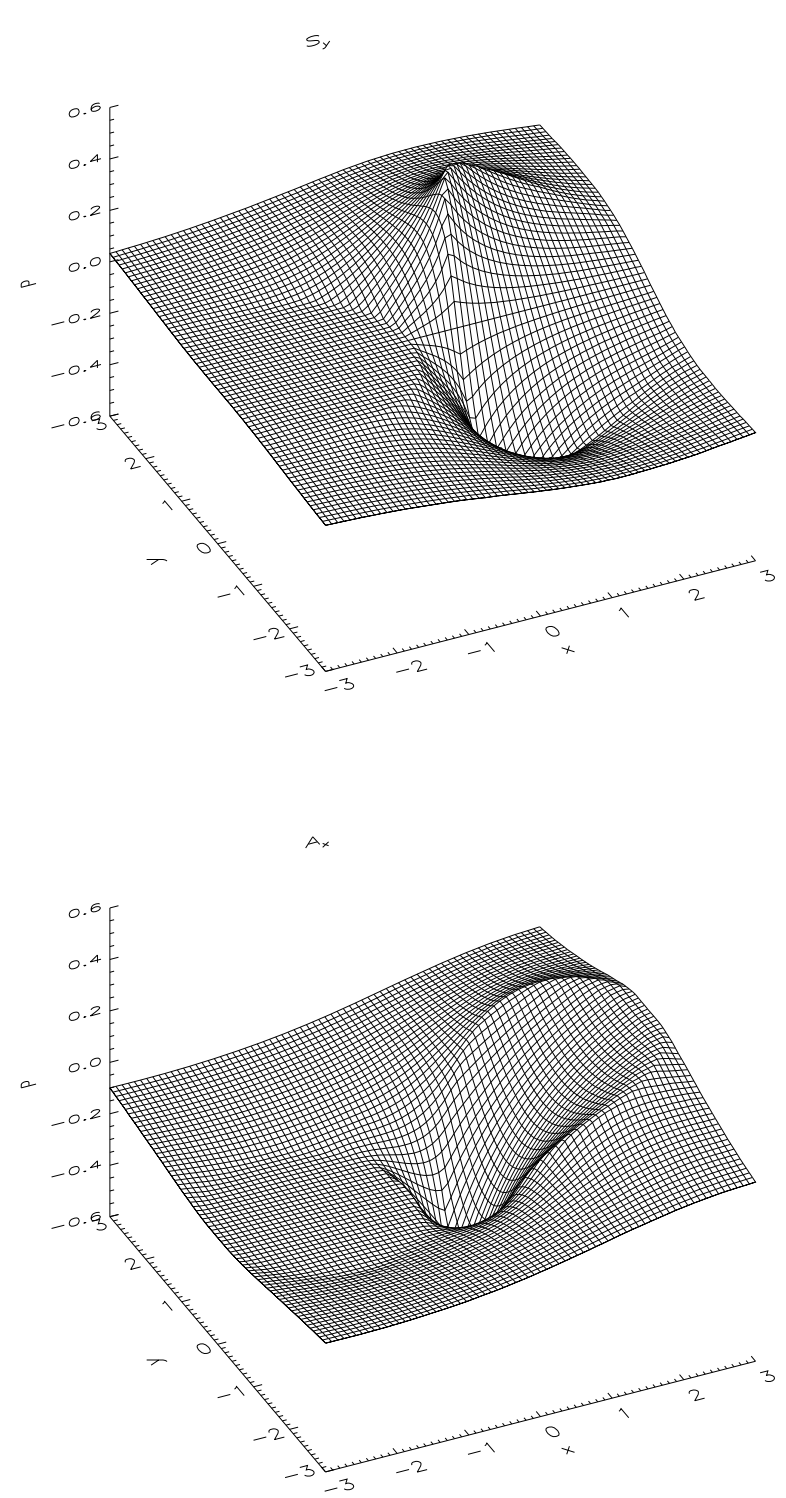

Fig. 5. The pressure eigenfunctions for $\zeta_{\mathrm{L}}=2, \zeta_{\mathrm{R}}=2.9$ and $\tau_{\mathrm{L}}=\tau_{\mathrm{R}}=$ $\ln 2$. The top panel is for $\sigma_{0}=\pi / 2$, whereas the bottom panel is for $\sigma_{0}=0$. The left tube does not respond to the oscillations of the right tube in either case.

parameters as in Fig. 4 and have taken $\tau_{0}=\ln 2$. This corresponds to an anomalous system: $\omega_{-}<\omega_{\mathrm{AL}}$. From the figure it is clear that the left tube does not respond to the oscillation of the right tube.

It can be proven that for systems where $\zeta_{L} \neq \zeta_{R}$, a critical separation between cylinders $d_{\mathrm{c}}$ can always be found so that the systems becomes anomalous for all $d<d_{\mathrm{c}}$.

\section{Eigenmodes of a system of two identical tubes}

In this section we consider a particular case when the two tubes are identical, i.e. when they have the same radii and the same internal plasma densities. We concentrate on $m=1$ and once again we simplify the notation by introducing $R=R_{\mathrm{L}}=R_{\mathrm{R}}$, $\rho_{i}=\rho_{\mathrm{L}}=\rho_{\mathrm{R}}, \zeta=\zeta_{\mathrm{L}}=\zeta_{\mathrm{R}}$, and $\tau_{0}=\tau_{\mathrm{L}}=\tau_{\mathrm{R}}$. Since the line $\zeta_{\mathrm{L}}=\zeta_{\mathrm{R}}$ is in the non-shaded sector in Fig. 3, a system of two identical loops always has the standard behaviour. 


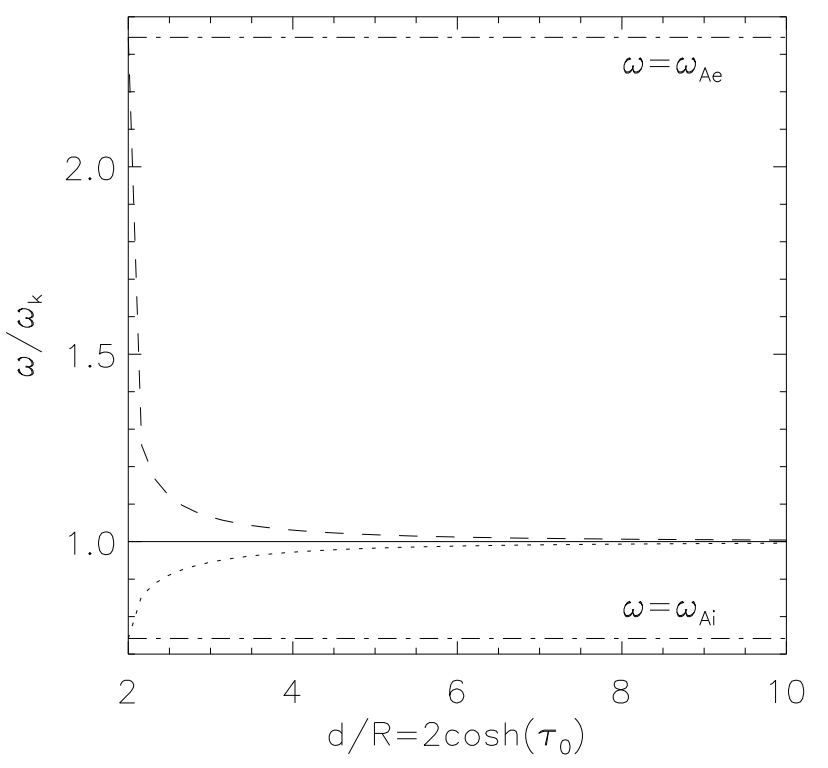

Fig. 6. The frequency normalised to the kink frequency of a single loop $\left(\omega / \omega_{k}\right)$ vs. the relative distance between the loops $(d / R)$. The dashed line shows $\omega_{+}$, while the dotted line shows $\omega_{-}$. The frequencies are calculated for $\zeta=10$. The dash-dotted lines indicate the internal, $\omega_{\mathrm{Ai}}$, and external, $\omega_{\mathrm{Ae}}$, Alfvén frequency, respectively.

The eigenmodes of the system of two identical tubes have been studied numerically by Luna et al. (2008). Because they used a different notation than ours, we fit the parameters that they used with tildes in order to compare our results. The loop radius in Luna et al. (2008) is $\tilde{a}$, so that $\tilde{a}=R$. Their parameter $\tilde{d}$ is defined as the distance between the loop centres, so that $\tilde{d}=$ $d=2 a \operatorname{coth} \tau_{0}$. Note a typographic error in their Eq. (3) where $\tilde{d}$ has been used instead of another parameter ( $a$ in our paper).

In the case of two identical tubes the dispersion relation (31) reduces to a very simple form,

$\omega_{ \pm}^{2}=\frac{2 V_{\mathrm{Ae}}^{2} k^{2}}{\zeta+1 \mp(\zeta-1) E}=\frac{\omega_{k}^{2}}{\left(1 \mp \frac{\zeta-1}{\zeta+1} E\right)}$,

where $E=\mathrm{e}^{-2 \tau_{0}}$. Recall that $\omega_{k}$ is the kink frequency given by $\omega_{k}=c_{k} k$. It follows from Eqs. (4) and (5) that $\cosh \tau_{0}=d / 2 R$, so that $\tau_{0}$ is a monotonically increasing and $E$ a monotonically decreasing function of the separation between the cylinders $d$. Then it follows that $\omega_{+}$is a monotonically decreasing and $\omega_{-}$ a monotonically increasing function of $d / 2 R$. This is in good agreement with the results reported by Luna et al. (2008).

A plot of the frequencies is shown in Fig. 6. The figure has been made with exactly the same parameters as Fig. 3 in Luna et al. (2008). As such, they can be directly compared to each other.

In the case of identical loops, the dependence of eigenfunctions on $\tau$ given by function $\Phi(\tau)$ takes an extremely simple form. For $\omega=\omega_{-}$, we obtain an odd function of $\tau$,

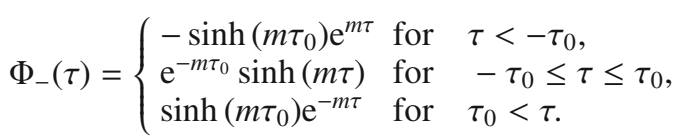

For $\omega=\omega_{+}$the function $\Phi(\tau)$ is even,

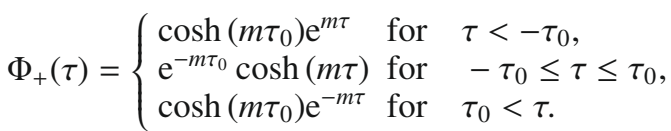

In Fig. 7 the pressure perturbation of the four eigenfunctions corresponding to kink oscillations of two identical loops is displayed. The displacement is calculated as the gradient of the pressure perturbation, so that Fig. 7 reproduces the kink mode structures.

When $d$ takes its minimum value, $d=2 R$, the cylinders touch each other. In this case

$\omega_{+}^{2}=\frac{\zeta+1}{2} \omega_{k}^{2}=\omega_{\mathrm{Ae}}^{2}, \quad \omega_{-}^{2}=\frac{\zeta+1}{2 \zeta} \omega_{k}^{2}=\omega_{\mathrm{Ai}}^{2}$,

where $\omega_{\mathrm{Ai}, \mathrm{e}}=V_{\mathrm{Ai}, \mathrm{e}} k$ is the Alfvén frequency inside/outside the loops. Once again these results agree very well with Luna et al. (2008). It is clear from their Fig. 3 that the frequencies of the $S_{x}$ and $A_{y}$ modes are very close to $\omega_{\mathrm{Ai}}$ for $d=2 R=2 \tilde{a}$. For the other branch, the limits are less clear. While the frequency of $A_{x}$ mode increases, probably to the external Alfvén frequency, the frequency of $S_{y}$ remains below the expected value for $d=2 R=2 \tilde{a}$.

As it has already been mentioned in Sect. 3, the main difference between our results and those obtained by Luna et al. (2008) is that, according to our analysis, the frequencies of the $S_{x}$ and $A_{y}$ modes coincide, and the same is true for the frequencies of the $S_{y}$ and $A_{x}$ modes. The first two frequencies are equal to $\omega_{-}$, and the last two to $\omega_{+}$. The most probable cause of this difference is that we neglected the wave dispersion related to the finite thickness of the system, so that, formally, our results are only valid in the limit $d / L \rightarrow 0$. In fact, the system numerically studied by Luna et al. (2008) is not thin at all. The largest differences between the frequencies of $S_{y}$ and $A_{x}$, and the frequencies of the $S_{x}$ and $A_{y}$ are observed for $\tilde{d} / \tilde{a}$ of the order of 3 . Since $\tilde{a}=0.1 L$, the differences between the frequencies take their maximum values for $\tilde{d} / L$ of the order of 0.3 , which is not small. However, even for this relatively thick system the difference between the frequencies of the $S_{x}$ and $A_{y}$ modes is smaller than $2 \%$. The difference between the frequencies of $S_{y}$ and $A_{x}$ is bigger with its maximum value of the order of $10 \%$. If our conjecture that the frequency differences are related to the wave dispersion is correct, then these differences should be very small for realistic values of parameters, $R=\tilde{a} \lesssim 0.02 L$.

To conclude this section, we notice an interesting analogy between the eigenmodes of the two-tube system and a single tube with an elliptic cross-section studied by Ruderman (2003). As we have already mentioned, when the separation distance takes its minimum value, $d=2 R$, the two loops touch each other. In this case, also formally the $A_{x}$ eigenmode still exists, it becomes unphysical because, to oscillate in anti-phase in the $x$-direction, the loops have to penetrate each other. The three other modes are still physical, but we concentrate on the $S_{x}$ and $S_{y}$ modes. For these two modes the system can be considered as a single magnetic loop consisting of two parallel magnetic tubes attached to each other. The size of the system in the $x$-direction is $4 R$, while it is $2 R$ in the $y$-direction. As we have seen, the oscillation frequency in the $x$-direction is lower than in the $y$-direction, so that the system oscillates with a lower frequency in the direction of its elongation. A similar result is valid for an elliptic loop: its oscillation frequency in the direction of the large axis is lower than that in the direction of the small axis.

\section{Summary and conclusions}

In this paper we have studied the eigenmodes of a system consisting of two parallel magnetic tubes. The ratios of loop radii to the separation distance and the ratios of the densities inside the 

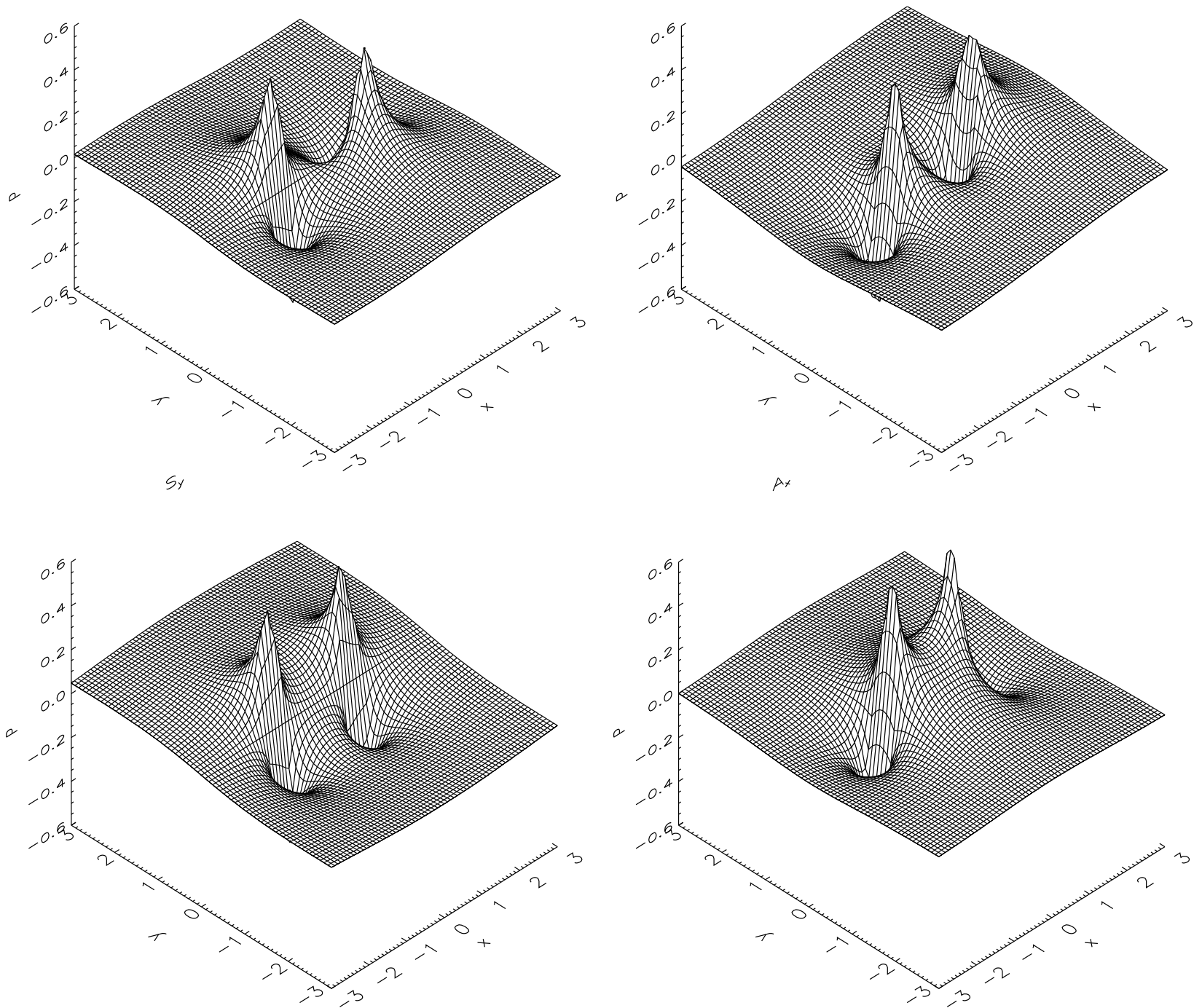

Fig. 7. The pressure perturbation for the eigenfunctions corresponding to kink oscillations $(m=1)$. Top left: $A_{y}\left(\omega=\omega_{-}, \sigma_{0}=\pi / 2\right)$, top right: $S_{x}$ $\left(\omega=\omega_{-}, \sigma_{0}=0\right)$, bottom left: $S_{y}\left(\omega=\omega_{+}, \sigma_{0}=\pi / 2\right)$, bottom right: $A_{x}\left(\omega=\omega_{+}, \sigma_{0}=0\right)$. The eigenfunctions were calculated for $\tau_{0}=2$, which corresponds to $d / R=7.52$.

loops to the external density are free parameters. We obtained the analytical expressions for the frequencies of two eigenmodes of the system oscillating in the directions connecting the centres of the tubes ( $x$-direction in what follows), and also the frequencies of two eigenmodes of the system oscillating in the perpendicular direction ( $y$-direction in what follows). We paid most attention to kink modes, and in what follows we only discuss the properties of these modes.

According to our analysis all kink eigenmodes can be divided into high-frequency eigenmodes with the oscillation frequency equal to $\omega_{+}$, and low-frequency eigenmodes with the oscillation frequency equal to $\omega_{-}$. There are two high- and two low-frequency eigenmodes. The first high-frequency eigenmode corresponds to antisymmetric oscillations in the $x$-direction, and is called the $A_{x}$ mode. The second high-frequency eigenmode corresponds to symmetric oscillations in the $y$-direction, and is called the $S_{y}$ mode.

Depending on the ratios of the densities in the left and right loops to the external density, $\zeta_{L}$ and $\zeta_{R}$, and the value of the quantity $\tau_{\mathrm{L}}+\tau_{\mathrm{R}}$ (see Eq. (4)), all systems can be divided in standard and anomalous systems. Standard systems are systems where the low frequency is above the internal Alfvén frequencies of the separate tubes. In anomalous systems, one tube has a higher Alfvén frequency than the eigenfrequency. In that case, the tube with the higher Alfvén frequency cannot support the collective oscillation.

In standard systems the first low-frequency eigenmode corresponds to symmetric oscillations in the $x$-direction, and is called the $S_{x}$ mode. The second low-frequency eigenmode corresponds to antisymmetric oscillations in the $y$-direction, and is called the $A_{y}$ mode. Hence, in standard systems there is one symmetric, $S_{x}$, and one antisymmetric, $A_{x}$, eigenmode of oscillation in the $x$-direction, and also one symmetric, $S_{y}$, and one antisymmetric, $A_{y}$, eigenmode of oscillation in the $y$-direction.

In anomalous systems the first low-frequency eigenmode corresponds to antisymmetric oscillations in the $x$-direction, and the second low-frequency eigenmode corresponds to symmetric oscillations in the $y$-direction. Hence, in these systems there are two $A_{x}$ modes, one high- and one low-frequency, and also two $S_{y}$ modes, one high- and one low-frequency. On the other hand there are no $S_{x}$ and $A_{y}$ modes. 
All systems of two identical tubes have a standard behaviour. As we have already mentioned, the frequencies of the $S_{x}$ and $A_{y}$ modes in standard systems are lower than those of the $A_{x}$ and $S_{y}$ modes, which agrees with a similar result found numerically by Luna et al. (2008).

We also found that the frequencies of the $S_{x}$ and $A_{y}$ modes in standard systems coincide, and the same is true for $A_{x}$ and $S_{y}$ modes. In anomalous systems the frequencies of highfrequency $A_{x}$ and $S_{y}$ modes coincide, and the same is true for low-frequency $A_{x}$ and $S_{y}$ modes. Since a linear combination of two eigenmodes corresponding to the same eigenfrequency is once again an eigenmode, this result implies that the system can oscillate in an arbitrary direction with two different frequencies. In this sense the system is degenerate. This result does not agree with Luna et al. (2008) who found that all four modes, $S_{x}, S_{y}$, $A_{x}$, and $A_{y}$, have different frequencies.

Our conjecture is that the system degeneration is related to the long-wavelength approximation that we used in our analysis. In this approximation we neglected the terms proportional to the square of the ratio of the tube separation distance to the tube length, $(d / L)$. We hope that continuing to the next order approximation with respect to the small parameter $(d / L)$ will remove the system degeneration. However, since $(d / L)$ is very small for realistic coronal structures, the difference between the frequencies of the $S_{x}$ and $A_{y}$ modes, and the frequencies of the $A_{x}$, and $S_{y}$ modes in standard systems will be very small. We also expect that the difference between the frequencies of the high-frequency $A_{x}$ and $S_{y}$ modes, and the frequencies of the lowfrequency $A_{x}$ and $S_{y}$ modes in anomalous systems will be very small.

Acknowledgements. This work was inspired by discussions at a workshop in Bern organised by ISSI. TVD would like to thank the hospitality of MSR and the Department of Applied Mathematics, University of Sheffield. This work was made with the support of STFC.

\section{References}

Andries, J., Arregui, I., \& Goossens, M. 2005a, ApJ, 624, L57

Andries, J., Goossens, M., Hollweg, J. V., Arregui, I., \& Van Doorsselaere, T. 2005b, A\&A, 430, 1109

Arregui, I., Van Doorsselaere, T., Andries, J., Goossens, M., \& Kimpe, D. 2005, A\&A, 441, 361

Aschwanden, M. J., Fletcher, L., Schrijver, C. J., \& Alexander, D. 1999, ApJ, 520,880

Aschwanden, M. J., Nightingale, R. W., \& Alexander, D. 2000, ApJ, 541, 1059

De Moortel, I., \& Brady, C. S. 2007, ApJ, 664, 1210

Dymova, M. V., \& Ruderman, M. S. 2005, Sol. Phys., 229, 79

Dymova, M. V., \& Ruderman, M. S. 2006a, A\&A, 457, 1059

Dymova, M. V., \& Ruderman, M. S. 2006b, A\&A, 459, 241

Edwin, P. M., \& Roberts, B. 1983, Sol. Phys., 88, 179

Erdélyi, R., \& Fedun, V. 2007, Science, 318, 1572

Erdélyi, R., \& Verth, G. 2007, A\&A, 462, 743

Goossens, M., Andries, J., \& Aschwanden, M. J. 2002, A\&A, 394, L39

Korn, G., \& Korn, T. 1961, Mathematical Handbook for Scientists and Engineers (New York: McGraw-Hill)

Luna, M., Terradas, J., Oliver, R., \& Ballester, J. L. 2008, ApJ, 676, 717

McEwan, M. P., Díaz, A. J., \& Roberts, B. 2008, A\&A, 481, 819

Nakariakov, V. M., \& Ofman, L. 2001, A\&A, 372, L53

Nakariakov, V. M., Ofman, L., DeLuca, E. E., Roberts, B., \& Davila, J. M. 1999, Sci., 285, 862

Ruderman, M. S. 2003, A\&A, 409, 287

Ruderman, M. S. 2007, Sol. Phys., 246, 119

Ruderman, M. S., \& Roberts, B. 2002, ApJ, 577, 475

Ryutov, D. D., \& Ryutova, M. P. 1976, Sov. J. Exp. Theor. Phys., 43, 491

Terradas, J., Oliver, R., \& Ballester, J. L. 2006a, ApJ, 642, 533

Terradas, J., Oliver, R., \& Ballester, J. L. 2006b, ApJ, 650, L91

Tomczyk, S., McIntosh, S. W., Keil, S. L., et al. 2007, Science, 317, 1192

Van Doorsselaere, T., Andries, J., Poedts, S., \& Goossens, M. 2004a, ApJ, 606, 1223

Van Doorsselaere, T., Debosscher, A., Andries, J., \& Poedts, S. 2004b, A\&A, 424, 1065

Van Doorsselaere, T., Nakariakov, V. M., \& Verwichte, E. 2007, A\&A, 473, 959

Van Doorsselaere, T., Nakariakov, V. M., \& Verwichte, E. 2008, ApJ, 676, L73

Verwichte, E., Nakariakov, V. M., Ofman, L., \& Deluca, E. E. 2004, Sol. Phys., 223, 77

Zaitsev, V. V., \& Stepanov, A. V. 1975, Issled. Geomagn. Aeron. Fiz. Solntsa, 3 